\title{
Unrelenting Lower Back Pain in an Uncontrolled Diabetic Patient With an Uncommon Diagnosis
}

\author{
Chad J. Cooper, Sarmad Said ${ }^{\mathrm{a}, \mathrm{b}}$, Sayeed Khalillullah, \\ Sucheta Gosavi ${ }^{a}$,Ogechika Alozie ${ }^{a}$
}

\begin{abstract}
Pyogenic spondylodiscitis is an uncommon but important infection that represents approximately $3-5 \%$ of all cases of osteomyelitis. Staphylococcus aureus is the most frequent causative microorganism that accounts for half of the cases of pyogenic spondylodiscitis. Arachnoiditis is a neuropathic disease with chronic inflammation of the arachnoid layer of the meninges that results in a very debilitating condition. This inflammation can trigger fibrous exudates, leading to the formation of scar tissue that causes the nerve roots to adhere to themselves and or the thecal sac. MRI is the modality of choice for imaging of spondylodiscitis and arachnoiditis due to its high sensitivity and specificity. The patient is a 60 years old female with the complaint of lumbar area back pain for five days that was constant, severe, dull in quality, radiated down legs and was aggravated by movement. Past medical history included uncontrolled diabetes and morbid obesity. The initial blood cultures were positive for methicillin sensitive staphylococcus aureas (MSSA). MRI of lumbar spine showed L4-L5 infectious spondylodiscitis, diffuse lumbosacral infectious arachnoiditis and a right psoas pyomyositis and $7 \mathrm{~mm}$ abscess. A PICC line was placed so that the patient could receive an eight week course of ceftriaxone $2,000 \mathrm{mg}$ IV daily. The usual causes of arachnoiditis are infection, spinal surgery, and intraspinal injection of steroid or myelography dye and spinal anesthesia. Spinal arachnoiditis can be diagnosed by myelography, computed tomography, and by magnetic resonance imaging (MRI) in combination with clinical symptoms and history taking. Arachnoiditis is a chronic disorder, with no known cure. It is very important for physicians to consider spondylodiscitis with arachnoiditis in their differential diagnosis when an uncontrolled diabetic patient presents with unrelenting back pain. Much consideration is needed to determine whether a MRI of the spine is needed earlier in the diagnostic process of lower back pain if the etiology seems to be of
\end{abstract}

\footnotetext{
Manuscript accepted for publication July 5, 2013

${ }^{a}$ Department of Internal Medicine, Texas Tech University Health Science Center, 4800 Alberta Ave, El Paso, 79905, El Paso, Texas, USA

${ }^{\mathrm{b}}$ Corresponding author: Sarmad Said, Department of Internal Medicine, Texas Tech University Health Science Center, 4800 Alberta Ave, El Paso, 79905, El Paso, Texas, USA. Email: sarmad.said@ttuhsc.edu
}

doi: http://dx.doi.org/10.4021/jmc1401w infectious nature.

Keywords: Arachnoiditis; Spondylidiscitis; Atypical back pain

\section{Introduction}

Pyogenic spondylodiscitis (PS) is an uncommon infection representing approximately 3 - 5\% of all osteomyelitis cases [1]; male-to-female ratio 3:1. PS occurs commonly from hematogenous speeding. It typically involves disc and anterior corners of the adjacent vertebral bodies.

Staphylococcus aureus is the most frequent microorganism accounting for half of the cases. Gram-negative rods account for $7-33 \%$ and coagulase-negative staphylococci were reported in $5-16 \%$ of cases [2]. The incidence has increased recently due to a more elderly population, chronic use of steroids and other comorbidities [3]. The disease is characterized by unremitting back pain. When the clinical presentation is suggestive, blood cultures should be performed. Magnetic resonance imaging is the modality of choice due to its high sensitivity and specificity.

Arachnoiditis is a neuropathic disease with chronic inflammation of the meningeal arachnoid layer. This inflammation triggers fibrous exudates, leading to the formation of scar tissue causing the nerve roots to adhere to themselves and/or the thecal sac [4]. Adhesive arachnoiditis is the endstage-inflammatory-process. The marked pia-arachnoid proliferation with dense collagen deposition produces complete nerve root encapsulation [5]. This causes compression of the nerve roots leading to a decrease in blood flow and malfunctioning. It most commonly occurs in the lower back but can spread up the spine. The extensive scarring of the meninges leads to burning and stabbing pain. While arachnoiditis has no consistent pattern of symptoms, it frequently affects the nerves that supply the legs and lower back. Many sufferers are unable to sit for long periods, and have difficulties controlling their limbs. The pain is typically not relieved with medications. Other symptoms that tend to accompany arachnoiditis include muscle spasms, weakness, numbness and tingling sensation of the extremities and bladder, bowel or sexual dysfunction. 


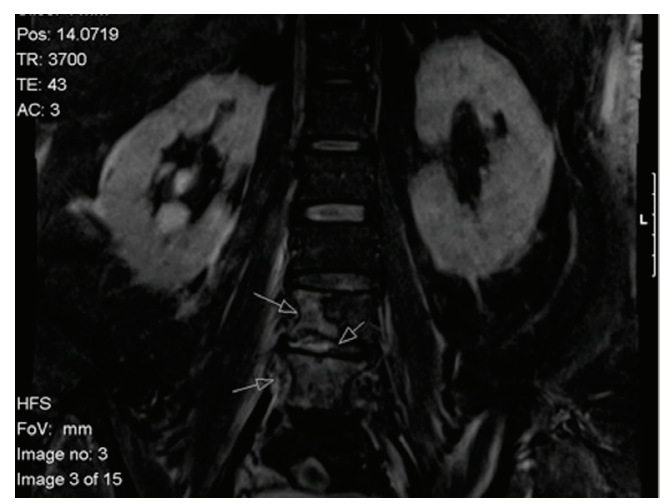

Figure 1. MRI T2 coronal view of lumbar spine: abnormal T2 hyperintensity and faint enhancement of L4-L5 intervertebral disc, with bone marrow edema seen within L4-L5 contiguous endplates and $3 \mathrm{~mm}$ anterior epidural fluid collection posterior to L4-5 intervertebral disc. The findings are consistent with L4-L5 infectious spondylodiscitis.

\section{Case Report}

The patient is a 60 -year-old female with five days complaint of constant, severe and dull lumbar pain, radiated to the legs and aggravated by movement; other complaints included fatigue, confusion, fever and chills. Past medical history was significant for uncontrolled diabetes and hypertension. Social history was unremarkable. On admission, she was hypertensive, tachycardic and febrile. Pertinent physical findings included confusion, mild respiratory distress and moderate tender to palpation at lower back. The remainder of the physical examination was negative.

The initial laboratory workup was remarkable for mild thrombocytopenia, mild hyponatremia, hyperglycemia (411 $\mathrm{mg} / \mathrm{dL}$ ) and elevated glycohemoglobin (A1c) (12.6\%); other values were normal. The initial blood culture was positive for methicillin-sensitive staphylococcus aureas (MSSA). Urine culture was negative.

Computer tomography (CT) of the brain was normal. CT of the chest showed bibasilar subsegmental atelectasis, multiple subcentimeter paratracheal lymph nodes, and multiple calcified lymph nodes in the right hilar region. CT of the abdomen and pelvis was normal. The x-ray of Lumbar spine showed minimal osteoarthropathic and discopathic changes of lower area. Endocarditis was ruled out echocardiographically. As demonstrated in Figure 1 the MRI of lumbar spine showed L4-L5 infectious spondylodiscitis. Figure 2 shows MRI T2 sagittal view of lumbar spine resembling a diffuse lumbosacral infectious arachnoiditis, right psoas pyomyositis and $7 \mathrm{~mm}$ abscess.

After four days of daily intravenous vancomycin 1,000 $\mathrm{mg}$, azithromycin $500 \mathrm{mg}$ and ceftriaxone $1,000 \mathrm{mg}$ the repeated blood cultures were negative. The infectious disease team was consulted. The previous antibiotic regimen was changed to naficillin 2,000 mg every 4 hours. Interventional

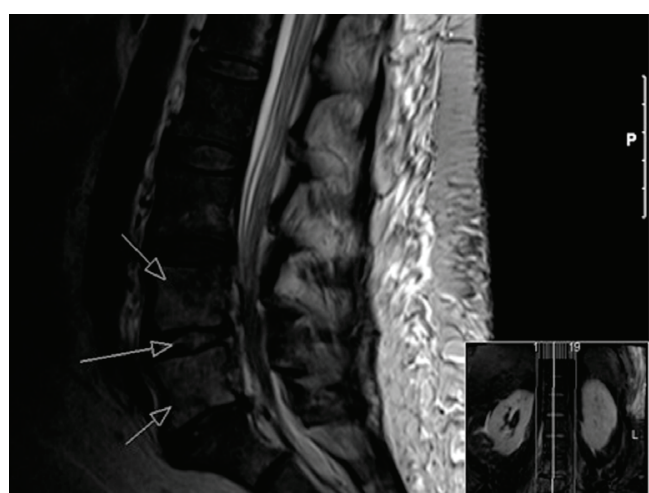

Figure 2. MRI T2 sagittal view of lumbar spine: abnormal diffuse leptomeningeal enhancement is identified along the distal spinal cord, conus medullaris, and entire cauda equine which is indicative of infectious arachnoiditis involving the lower thoracic and entire lumbosacral segments.

radiology was not able to drain the right psoas abscess due to its small size. However, a peripherally-inserted-central-line was placed so that the patient could receive an eight-week course of daily ceftriaxone $2,000 \mathrm{mg}$. She was discharged on the ninth day.

\section{Discussion}

The symptomatology of arachnoiditis correlates with the extent and type of nerve root involvement, the inflammation stage, the magnitude and persistence of mechanical and chemical insults [6]. The initial stage is characterized by radiculopathy caused by inflammation of the pia-arachnoid mater with nerve root swelling. Adhesive arachnoiditis represents the resolution of the inflammatory process and dense collagen deposition causing complete encapsulation of nerve roots, which then undergo progressive atrophy [7]. The causes of arachnoiditis are infection, spinal surgery, intraspinal injection and spinal anesthesia [8]. Myelograms with injection of contrast have been described as a chemically induced cause.

Arachnoiditis can be caused by certain infections including syphilis, meningitis, tuberculosis, staphylococcus aureus and candida [9]. Spinal arachnoiditis can be diagnosed by myelography, CT and by MRI in combination with clinical symptoms. The appearance of arachnoiditis on MRI depends on the inflammation and scarring severity. One pattern is a central clump (single or multiple) of nerve roots within the spinal canal. The second pattern, wherein roots adhere to the peripheral walls of the thecal sac, is called the "empty sac" pattern: the spinal canal appears devoid of nerve roots [10]. The third and least-common pattern is of a single, large, nonspecific-appearing soft tissue mass within the spinal canal. An electromyogram can assess the severity of the ongoing damage to affected nerve roots by using electrical impulses. 
Arachnoiditis is a chronic disorder, with no known cure. Palliation is achieved to some degree by the use of analgetics and increasingly long periods of sitting or lying flat [11]. Physical therapy such as hydrotherapy, massage, hot or cold therapy or transcutaneous electrical nerve stimulation provides some relief. Treatment of spinal arachnoiditis is difficult for the physician and the patient, because complete pain relief remain impossible in most cases; the prognosis is poor. Surgical intervention can provide only temporary relief.

Lower back pain is a very common cause for visit to a physician that affects approximately $80 \%$ of the population at some point during their lives. The majority of lower back pain results from injuries; but it can also result from other diseases. It is very important to consider spondylodiscitis with arachnoiditis in the differential diagnosis when an uncontrolled diabetic patient presents with unrelenting back pain.

\section{Conclusion}

The usual causes of arachnoiditis are infection, spinal surgery, and intraspinal injection of steroid or myelography dye and spinal anesthesia. Spinal arachnoiditis can be diagnosed by myelography, CT and MRI. It is a chronic disorder, without known cure. It is essential for physicians to consider it in the differential diagnosis when an uncontrolled diabetic patient presents with unrelenting back pain. Much consideration is needed to determine whether a MRI of the spine is needed earlier.

\section{Disclosures}

All participated authors in this study declare no financial, professional or personal conflicts.

\section{References}

1. Fantoni M, Trecarichi EM, Rossi B, Mazzotta V, Di Giacomo G, Nasto LA, Di Meco E, et al. Epidemiological and clinical features of pyogenic spondylodiscitis. Eur Rev Med Pharmacol Sci. 2012;16(Suppl 2):2-7.

2. Sans N, Faruch M, Lapegue F, Ponsot A, Chiavassa H, Railhac JJ. Infections of the spinal column--spondylodiscitis. Diagn Interv Imaging. 2012;93(6):520-529.

3. Bettini N, Girardo M, Dema E, Cervellati S. Evaluation of conservative treatment of non specific spondylodiscitis. Eur Spine J. 2009;18(Suppl 1):143-150.

4. Wright MH, Denney LC. A comprehensive review of spinal arachnoiditis. Orthop Nurs. 2003;22(3):215-219; quiz 220-211.

5. Burton CV. Lumbosacral arachnoiditis. Spine (Phila Pa 1976). 1978;3(1):24-30.

6. Quiles M, Marchisello PJ, Tsairis P. Lumbar adhesive arachnoiditis. Etiologic and pathologic aspects. Spine (Phila Pa 1976). 1978;3(1):45-50.

7. Ransford AO, Harries BJ. Localised arachnoiditis complicating lumbar disc lesions. J Bone Joint Surg Br. 1972;54(4):656-665.

8. Na EH, Han SJ, Kim MH. Delayed occurrence of spinal arachnoiditis following a caudal block. J Spinal Cord Med. 2011;34(6):616-619.

9. Dolan RA. Spinal adhesive arachnoiditis. Surg Neurol. 1993;39(6):479-484.

10. Ross JS, Masaryk TJ, Modic MT, Delamater R, Bohlman H, Wilbur G, Kaufman B. MR imaging of lumbar arachnoiditis. AJR Am J Roentgenol. 1987;149(5):10251032.

11. Bourne IH. Lumbo-sacral adhesive arachnoiditis: a review. J R Soc Med. 1990;83(4):262-265. 